\title{
The rat's long-term preference for complexity in its environment
}

\author{
MICHAEL S. DENNY \\ Michigan State University, East Lansing, Michigan 48824
}

\begin{abstract}
Locomotor, resting, and feeding responses to four levels of environmental complexity were measured in a two-phase study. In a long-term free-choice testing situation (ad-lib phase), subjects $(N=20)$ demonstrated a marked preference for high complexity during "day" conditions. This preference, present in the patterns of resting and locomotor behavior, was partially preserved under "night" conditions. The patterns of feeding behavior were essentially independent of complexity preference. The same subjects in the discrete trial phase of the study chose high complexity most often in a four-choice testing situation. The apparent preference for high environmental complexity was interpreted as a shelter/relaxation seeking response elicited by the number or complexity of elements in the complexity area. Complexity seemed to reduce the arousal level of the subject, arguing against current arousal hypotheses which suggest that complex stimuli are attractive because of their arousal-inducing qualities.
\end{abstract}

The effect of the structure or complexity of an animal's environment on its behavior has been a recurring theme in both motivational and ethological research. Usually the variety or density of some set of elements extracted from the environment has operationally determined its complexity. In a number of early studies, the attention paid to preference responding toward quantitatively distinct levels of environmental complexity reflected a basic concern with the motivational underpinnings of exploratory behavior (Berlyne, 1955; Dember \& Earl, 1957; Montgomery, 1955).

Preference responding to environmental displays has also been used as a measure of an animal's proclivity to approach and remain in ecologically appropriate environments (Harris, 1952; Klopfer \& Hailman, 1965; Sale, 1969). In laboratory studies of the rat, the ethological relevance of complexity has been suggested by its relationship to feeding, grooming, and exploration (Bindra \& Spinner, 1958; Hughes, 1968; Pereboom, 1968), although none of these studies attempted to articulate the relationships.

The motivational and the majority of the ethological studies of environmentally affected behavior have relied on the short-term assessment of a few dependent variables (usually locomotor) and have not considered the influence of basic adjunctive factors such as diurnal phase. The present experiment investigated the long-term behavior of rats in familiar environments which differed in the number and variety of three-dimensional elements. Controlled illumination periods of "day" and "night" were used to induce different "arousal" conditions under which complexity preference was examined. The preference response was treated as a behavioral syndrome; and a number of responses, consummatory as well as appetitive, were

I would like to thank Ralph Levine, my thesis advisor, for his guidance and support. measured. This approach allowed a comparison as to how and to what extent different areas of complexity were used by the animal.

\section{METHOD}

\section{Subjects}

Twenty male nonsibling Sprague-Dawley rats, $70-80$ days old at the beginning of the experiment, were individually housed and run.

\section{Apparatus}

The experimental cages were $1.22 \times 2.44 \times .61 \mathrm{~m}$ wooden frames covered on all sides with hardware cloth. The cages were divided into four equal-sized compartments by masonite panels joined to a small (22-cm square) center section. A clear Plexiglas door was positioned at floor level in each of the hardware cloth walls of the center section. The doors allowed access to any compartment from any other compartment through the center section.

Complexity was determined by fixing one of four inserts in a compartment. The inserts were simple lattice frames $(.56 \times 1.16 \mathrm{~m})$ with lengths of chain attached to one face. An insert lattice was fixed horizontally to brackets along the top of the cage walls forming a false-ceiling with chains extending down to the compartment floor. Four complexity levels were created using three types of chain distributed in the following combinations and densities: open complexity-no chains; low complexity -9 bead chains; medium complexity -9 bead chains and 13 furnace chains; high complexity-9 bead chains, 13 furnace chains, and 13 tensor-link chains. The 46-cm lengths of chain had maximum diameters of $3 \mathrm{~mm}$ (bead), $9 \mathrm{~mm}$ (furnace), and $16 \mathrm{~mm}$ (tensor link). When the inserts were in place, the overall incident light levels in the four compartments were computed to differ by no more than $.5 \%$.

Movement within a compartment was detected by eight $10 \times 12.7 \mathrm{~cm}$ sensor plates distributed across its floor. The sheet metal plates served as momentary contact switches which were activated when the subject stepped on the plate. One of the plates was positioned in front of the food-hopper/water-bottle fixture that was attached to the outside of each compartment's end wall. To another plate, a four-sided aluminum box was attached, creating a $10 \times 12.7 \times 10 \mathrm{~cm}$ "resting box" open at one end and positioned near the same wall (diagonally across from the Plexiglas door to the center section). The remaining six 
Table 1

Twelve-Hour Day and Night Means of the Ad Lib Phase Dependent Measures Including Rate Scores

\begin{tabular}{|c|c|c|c|c|c|c|c|c|}
\hline \multirow[b]{2}{*}{ Score } & \multicolumn{4}{|c|}{ Day } & \multicolumn{4}{|c|}{ Night } \\
\hline & High & Medium & Low & Open & High & Medium & Low & Open \\
\hline Occupancy Time & 373.4 & 82.2 & 83.2 & 12.7 & 233.4 & 138.0 & 146.2 & 71.8 \\
\hline Resting Time & 265.8 & 71.1 & 65.1 & 9.1 & 71.3 & 18.4 & 34.6 & 18.6 \\
\hline Feeding Time & .889 & .278 & .778 & .264 & 6.32 & 6.59 & 7.15 & 5.71 \\
\hline Entry Frequency & 2.28 & 1.83 & 1.64 & .75 & 21.1 & 18.9 & 18.8 & 15.9 \\
\hline Locomotor Activity & 10.78 & 5.47 & 6.55 & 3.39 & 100.1 & 83.2 & 80.5 & 76.0 \\
\hline Food Consumption & .861 & .861 & 1.083 & .403 & 5.65 & 5.11 & 3.99 & 4.04 \\
\hline Water Consumption & 2.47 & 1.94 & 2.42 & 2.05 & 7.81 & 9.18 & 9.29 & 6.22 \\
\hline Defecation & 2.05 & 1.22 & 1.61 & .33 & 5.69 & 7.08 & 5.03 & 6.06 \\
\hline Food and Water Consumption Rate & & & & & .113 & .163 & .151 & .218 \\
\hline Defecation Rate & & & & & .0369 & .0706 & .0418 & .1275 \\
\hline Locomotor Activity Rate & & & & & .952 & 1.079 & .907 & 1.863 \\
\hline
\end{tabular}

plates were quasirandomly distributed across the compartment floor.

Movement between compartments was detected by momentary contact switches integral with the hinges at the top of each Plexiglas door. Two 20-channel Esterline Angus event recorders, operated in tandem, recorded activations of the floor plates and door switches.

\section{Procedure}

Subjects were initially habituated to the experimental room in individual rack cages for 5 days. During this holding time, with free access to food (Purina Lab Chow) and water, the animals were handled once daily for about $1 \mathrm{~min}$.

The holding cages and the experimental cages were located in two light-proofed rooms. All lights, two $100-\mathrm{W}$ bulbs $1.5 \mathrm{~m}$ above each cage, were automatically switched on at $8 \mathrm{a} . \mathrm{m}$. and off at $8 \mathrm{p} . \mathrm{m}$. Before an experimental session, the cages were washed and the four different complexity inserts were quasirandomly assigned to the four compartments.

Ad lib phase. At $8 \mathrm{a} . \mathrm{m}$. on the morning following the 5 th day of habituation to the room environment, the subject was placed in the center section of the experimental cage. Food and water supplies were maintained in all compartments. The recorders were turned on after $120 \mathrm{~h}$ of occupancy. Subsequently, food and water consumption and defecation were measured every $12 \mathrm{~h}$. At these times, food and water supplies were replenished, and the litter trays underneath each compartment were cleaned.

During the final $48 \mathrm{~h}$, data were collected separately for each compartment and eventually converted into the following 12-h scores: (1) occupancy time (total time in minutes within compartment); (2) locomotor activity (total number of discrete depressions of all floor plates); (3) feeding time (total time in minutes that floor plate in front of food and water containers was depressed); (4) resting time (total time in minutes floor plate at bottom of rest box was depressed); (5) entry frequency (number of entries made into compartment); (6) food consumption (in grams); (7) water consumption (in grams); (8) defecation (number of fecal boli).

The subject was removed from the cage and returned to its holding cage at the end of $168 \mathrm{~h}$ of occupancy. The first $120 \mathrm{~h}$ served as a period of habituation (to the experimental cage) intended to eliminate the effects of novelty and stabilize responding in this and the following phase of the experiment.

Discrete trial phase. At about 8 p.m. on the day of removal from the experimental cage, and then every $12 \mathrm{~h}$ for a total of six sessions, the subject was given three discrete testing trials. At the beginning of a session, the subject was transported in its holding cage to the same experimental cage used for the ad lib phase. It was placed in the center section of the cage and, once it had entered one of the compartments, the door was blocked to prevent subject's retreat. After $3 \mathrm{~min}$, the subject was removed from the compartment and returned to its holding cage. Two more trials were given with 30 -min ITIs and the subject was returned to the holding cage rack until the next session. Thus, over a 60 -h span, the subject received 18 discrete testing trials.

All discrete trial testing was carried out under day illumination which required extending the light period to about 9 p.m. During testing, the recorders were operative, and the data were eventually converted into the following scores for each compartment: (1) weighted choice (number of entires on all trials across all sessions multiplicatively weighted by the number of entries on all first trials across all sessions); (2) mean entrance latency (time in seconds spent in the center section); (3) mean locomotor activity (number of discrete depressions of all floor plates).

\section{RESULTS}

The experimental design was treated as a 4 by 2 (complexity by light period) factorial with repeated measures. Unless stated otherwise, the statistics presented are from ANOVAs. All differences between complexity levels were tested using Tukey's procedure for post hoc paired comparison (Winer, 1962) at the .05 alpha level.

\section{Ad Lib Phase}

As expected, general activity was highly attenuated during the day. For example, the mean entry frequency into all compartments per day was 6.00 , as compared to 74.72 per night $[F(1,17)=257.92, p<.0005]$. Two subjects that spent the total $48 \mathrm{~h}$ in one compartment were not included in any analysis. 1 The distribution of general activity (locomotor activity, entry frequency, etc.) was distinguished by peaks immediately following changes in the light period, suggesting an excitatory effect of the change in environmental state and by peaks during the 5th and the 12th hours of darkness.

The overall response to complexity, independent of light period, was characterized by differential occupancy and resting times $[F(3,51)=11.93$ and 8.46 , respectively; $\mathrm{p}<.0005]$ as well as differences in locomotor activity and entry frequencies $[F(3,51)=$ 3.64 and 4.05 , respectively; $p<.02$ ). The separate day and night means of the eight scores across complexity levels are presented in Table 1 . The data clearly show that subjects spent most resting and nonresting occupancy time in the high complexity compartment, with these times falling off rather systematically at lower 

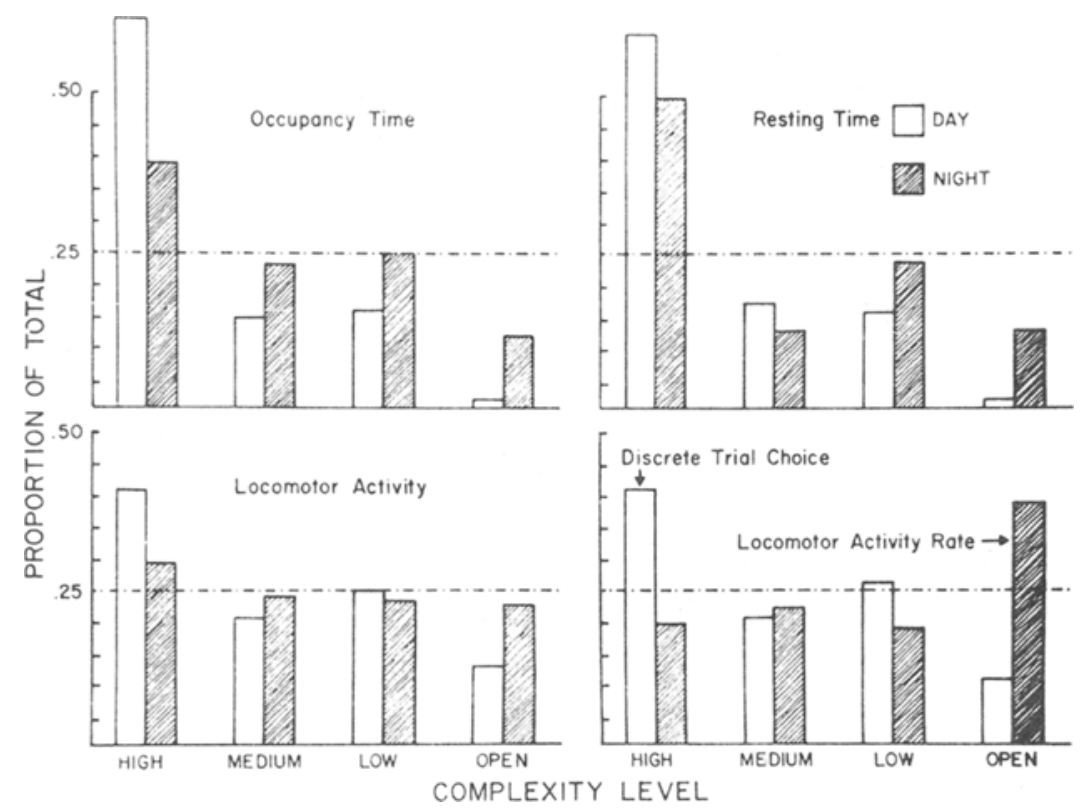

Figure 1. Response to complexity for selected day and night ad-lib measures, discrete trial choice, and night ad-lib locomotor activity rate. levels. Although this was true for both day and night behavior, the result was more marked under day illumination. Locomotor activity and entry frequency followed the same pattern.

These four scores were significantly greater for high complexity vs. all other levels under day and night conditions, except entry frequency where high was only greater than open complexity during the night. In addition, daytime behavior revealed significantly lower scores for open vs, all other levels in occupancy time and entry frequency measures. All differences between the low and medium means lacked significance. These comparisons support the notions that the scores were: (1) positively and monotonically related to complexity level, (2) primarily sensitive to complexity contrasts involving the high level and secondarily sensitive to contrasts involving the open level, and (3) more differentiated under day illumination.

The remaining scores (feeding time, food and water consumption, defecation) were unaffected by complexity level [all $F(3,51) s<1.0$ ]. However, when these scores were converted to rates by dividing the nighttime value of the score by the corresponding active occupancy time (occupancy time minus resting time), significant complexity effects were found (see Table 1 ). The nighttime pattern across complexity levels was in contrast to scores such as occupancy time, revealing a monotonically negative relationship between rate of performance and complexity.

The nighttime food and water consumption rate (combined score) varied significantly with complexity $[F(3,51)=5.53, p<.003]$ being highest in open complexity and lowest in high complexity. The nighttime defecation rate $[F(3,51)=6.87, p<.001]$ was also highest in open complexity. In addition, the rate conversion was made on the nighttime locomotor activity score. Activity rate showed a similarly significant variance $[F(3,51)=8.71, p<.0005)$ being highest in open complexity. Nighttime activity rate is compared to the contrasting day and night patterns of some selected ad lib scores in Figure 1. The proportion of the total incidence of the behavior that occurred in each complexity compartment is represented by bar height. The dotted horizontal line indicates the proportion expected by chance.

A significant interaction effect between complexity and illumination period, for occupancy and resting times $[F(3,51)=7.08$ and 8.75 , respectively; $p<.0005]$, can be seen in the figure. The nighttime shift in the response pattern was characterized by reduced time spent in high complexity, relative to all other levels, and an increase in the relative resting time in open complexity.

A correlational analysis of all scores indpendent of complexity level was made to assess the general behavior pattern demonstrated by subjects' total multivariate response to the experimental cage. The Pearson product moment correlations are given separately for day and night results in Table 2.

The sequence of entry behavior within the illumination period intervals was also considered. The distribution of entries made from a compartment (i.e., the conditional probabilities) was compared to the expected values weighted to correspond to the unequal distribution of total entries into the four compartments. The resulting $\chi^{2}$ values reflected the degree to which a preference for visiting a particular complexity level was determined by the immediate history of the last compartment occupied. During the day, exits from the low complexity compartment significantly favored the high complexity compartment $\left(\chi^{2}=13.99, \mathrm{df}=2\right.$, 
Table 2

Correlations Between Ad Lib Scores Lnder Day (Left-Hand Matrix) and Night (Right-Hand Matrix) Conditions including the Discrete Trial Choice Score

\begin{tabular}{|c|c|c|c|c|c|c|c|c|c|}
\hline & OT & RT & $\mathrm{FT}$ & EF: & LA & lic & $\mathrm{WC}$ & D & $\mathrm{C}$ \\
\hline Occupancy Time & - & .73 & .12 & .59 & .38 & .28 & .16 & .65 & .65 \\
\hline Resting Time & .89 & - & .04 & .41 & .32 & .08 & .12 & .39 & .58 \\
\hline Feeding Time & .25 & .04 & - & .34 & .55 & .62 & .41 & -.00 & -.06 \\
\hline Entry Frequency & .53 & .51 & .50 & - & .42 & .31 & .16 & .50 & .40 \\
\hline Locomotor Activity & .40 & .41 & .45 & .81 & - & .29 & .06 & .25 & .25 \\
\hline Food Consumption & .22 & .19 & .24 & .24 & .22 & - & .33 & .21 & .13 \\
\hline Water Consumption & .09 & .07 & -.04 & .20 & .22 & .10 & - & .16 & .21 \\
\hline Defecation & .48 & .50 & .12 & .38 & .17 & .34 & .08 & - & .36 \\
\hline Choice & .45 & .45 & .10 & .30 & .20 & .01 & .26 & .18 & - \\
\hline
\end{tabular}

$p<.001)$. This trend continued under night conditions $\left(\chi^{2}=6.53, \quad \mathrm{df}=2, \mathrm{p}<.05\right)$. Further, nighttime preference was shown for medium complexity when the subject was exiting from high complexity $\left(\chi^{2}=7.37\right.$, $\mathrm{df}=2, \mathrm{p}<.025)$. All other exit patterns demonstrated a lack of a specific complexity preference.

\section{Discrete Trial Phase}

Means of the three discrete trial scores are presented in Table 3. Only' the weighted choice measure was significantly affected by the environmental complexity dimension $[F(3,17)=4.97, p<.005)$. The weighting on this score emphasized first trial responding to counter the probability that subsequent trials within a session were influenced by recent exposures to other complexity levels. It was expected that this score would reflect long-term preference. The complexity level means were differentiated in a pattern similar to the ad lib results, with subjects choosing high complexity most often and open complexity least often. This trend is compared to the proportional distributions of selected ad lib scores in Figure 1. The Pearson correlations between discrete trial choice and the ad lib scores are included in Table 2.

\section{DISCUSSION}

The time the subject spent in any one compartment appears to be primarily a result of resting behavior, particularly during the day when the correlation between the occupancy time score and the resting time score was .89 , but also during the night (correlation of .73 ; see Table 2). In nature, it is quite rare to see animals sleeping or resting in the open. A rather obvious interpretation is that complexity, as chain density, is a critical component of shelter or hiding-place stimuli. Shelter, in the form of plant and rock cover, has been shown by Sale (1969) to be a major factor in the habitat

Table 3

Means of the Discrete Trial Dependent Measures

\begin{tabular}{lcccc}
\hline Score & High & Medium & Low & Open \\
\hline Weighted Choice & 22.4 & 11.4 & 14.3 & 5.8 \\
Latency & 6.94 & 8.11 & 7.29 & 7.91 \\
Locomotor Activity & 7.47 & 6.79 & 7.29 & 9.94 \\
\hline
\end{tabular}

preference of fish, to the exclusion of many presumably salient variabies.

The importance of shelter as the salient parameter of complexity preference may be seen in the loss of a strong differentiated response to complexity under night conditions where feeding activity and general locomotion characterized subjects' behavioral disposition. It can be concluded that the greater approach value associated with higher levels of complexity was primarily determined by their attractiveness for resting (or shelter) which was a high-probability response only under the day condition.

The same interpretation holds for the discrete trial performance if the testing situation is assumed to be slightly traumatic and it is remembered that daylight conditions prevailed. Here, the consequences of the approach response can be viewed as escape to an environment associated with shelter and relaxation. The consistently short latencies to enter a compartment supports the escape notion. This class of preference responding has been studied recently by Aitken (1974) who has shown that fearful (shocked) rats lose their novelty preference and choose familiar and dark surroundings. The fact that the discrete trial choice score correlated better with occupancy time of the first phase than with entry frequency, its ad-lib analogue, suggests that the discrete choice response and ad-lib occupancy time reflect the same consummatory nature of preference responding, that is, shelter/relaxation attainment.

Stressing the importance of the concomitant effects of a stimulus on the internal state of an organism, some investigators (e.g., Berlyne, 1960) have posited that higher animals tend to select levels of stimulus complexity which induce moderate amounts of arousal. In the present study, the relationship between complexity preference and arousal, though poorly pronounced in most cases, was essentially negative. In two general cases, when arousal was inferred from independent variable manipulations and from dependent variable performance, high arousal states led to undifferentiated responding or higher performance probabilities in the least complex surroundings. The obviously arousing shift to night illumination degrades 
the definition of any complexity preference yet does not produce a change in the rankings of the compartments. The most attractive compartment, in terms of approach and occupancy, evidences a high rate of resting which belies the notion that complexity is arousing the subject. Were arousal equated with activity rate, it would be found that arousal decreased monotonically with complexity. This observation contributes to a shelter/relaxation explanation while being problematic to current arousal hypotheses, at least for long-term behavior.

When short-term responding was considered, some support for the stimulus discrepancy interpretation (Dember \& Earl, 1957), sometimes incorporated by arousal hypotheses, was revealed. That is, there is evidence from the exit patterns that when the subject moved from one compartment to the next, it preferred one providing a moderate change in complexity level rather than one providing an extreme or a minimal change. This is not construed as antagonistic to the shelter/relaxation idea, but rather as an indication that separate preference behaviors are involved. In other words, when general activity is high, shelter seeking is subordinated in the short run by a tendency to seek environments (or stimulus levels) moderately discrepant from the currently occupied (and presumably adapted) environment. In a relatively chronic short-term (discrete trial) testing situation, Taylor (1974) reports that both incremental and decremental shifts from a well-adapted midcomplexity stimulus constitute effective incentives for approach, although the incremental shift is more effective.

In the present study, even when resting time is subtracted out, the high-complexity compartment garnered more occupancy time and entries than the other compartments. It appears likely that the exploratory incentive of these compartments increased with complexity. For periods of high locomotor activity, frequent entries into all compartments and short mean occupancy intervals (conditions assumed to indicate exploratory behavior), it was found that total activity monotonically increased with complexity. Although the differences were not as great as during periods of relative inactivity, and indeed because of this, they suggest that complexity offered a secondary incentive for exploration or approach. This incentive may be the factor responsible for the observed exit patterns where approach was an inverted " $U$ "-shaped function of stimulus discrepancy (i.e., the relative difference in complexity between two compartments). Similar short-term shifts in approach behavior have been reported by Dutch and Brown (1971) and May (1968).

The important finding of this study was that rats reacted to a relatively abstract form of environmental complexity in a manner consistent with ethological expectations of how an animal would exploit its natural habitat. Further, the findings suggest that some critical features of an animal's environment are perceived and responded to on the basis of their textural or pattern complexity. As in the present case, the biological impact of the environmental features is a mediated consequence of the complexity. That is, the selection of ecologically viable environments can depend on the eliciting value of apparently neutral abstractions of environmental features. Hilden (1965) has suggested the same relationship for neutral features in the natural environment.

The ecological component of responding to complexity may be an important source of variability when different species and different kinds of complexity are compared. It is possible that this component helps explain the diverse and often contrasting findings on complexity preference.

\section{REFERENCES}

Aitken, P. P. Aversive stimulation and rats' preference for areas differing in novelty-value and brightness. Animal Behaviour, $1974,22,731-734$.

Berlyne, D. E. The arousal and satiation of perceptual curiosity in the rat. Journal of Comparative and Physiological Psy chology, 1955, 48, 238-246.

Berlyne, D. E. Conflict, arousal, and curiosity. New York: McGraw-Hill, 1960.

Bindra, D., \& Spinner, N. Response to different degrees of novelty: The incidence of various activities. Journal of the Experimental Analy sis of Behavior, 1958, 1, 341-350.

Dember, W. N., \& Earl, R. W. Analysis of exploratory, manipulative, and curiosity behavior. Psychological Review, $1957,64,91-96$

Dutch, J., Brown, L. B. Visual stimulus complexity and approach behavior in rats. Psychonomic Science, 1971, 22, 162-163.

Harris, V. T. An experimental study of habitat selection by prairie and forest races of the deer mouse, Peromyscus maniculatus. Contributions of the Laboratory of Vertebrate Biology, University of Michigan, 1952, 56, 1-53.

Hilden. $O$. Habitat selection in birds, a review. Annales Zoologici Fennici, 1965, 2, 53-75.

Hughes, R. N. Behavior of male and female rats with free choice of two environments differing in novelty. Animal Behaviour, $1968,16,92-96$.

Klopfer, P. H., \& Hailman, J. P. Habitat selection in birds. In: S. Lehrmen, R. A. Hinde, and E. Shaw (Eds.), Advances in the study of behavior (Vol. 1). New York: Academic Press, 1965, 279-303.

May, R. B. Pretest exposure, changes in pattern complexity and choice. Joumal of Comparative and Phy siological Psy chology, $1968,66,139-143$.

Montgomery, K. C. The relations between fear induced by novel stimulation and exploratory behavior. Journal of Comparative and Physiological Psychology, 1955, 48, 132-136.

Pereboom, A. Systematic-representative study of spontaneous activity in the rat. Psy chological Reports, $1968,22,717-732$.

Taylor, G. T. Stimulus change and complexity in exploratory behavior. Animal Learning and Behavior, 1974, 2, 115-118.

Sale, P. F. A suggested mechanism for habitat selection by the juvenile manini acanthrus truostegus sandvicesis streets. Behavior, $1969,35,27-44$.

Winer, B. J. Statistical principles in experimental design. New York: MeGraw-Hill, 1962.

\section{NOTE}

1. There are incomplete data indicating that these two subjects remained for the entire 7 days of the ad lib phase in the first compartment they entered, high complexity for one and low complexity for the other. Upon removal from the experimental cage, these subjects exhibited extreme agitation and freezing behavior which was noted to persist throughout the discrete trial phase. 